Research Paper

\title{
Validation of the Prediction Model for Success of Vaginal Birth after Ce- sarean Delivery in Japanese Women
}

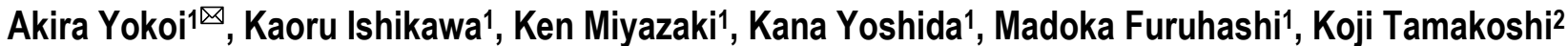

1. Department of Obstetrics and Gynecology, Japanese Red Cross Nagoya Daiichi Hospital, Nagoya, Japan;

2. Department of Nursing, Nagoya University School of Health Sciences, Nagoya, Japan.

$\triangle$ Corresponding author: Dr. Akira Yokoi, Department of Obstetrics and Gynecology, Japanese Red Cross Nagoya Daiichi Hospital, 3-35 Michishita-cho, Nakamura-ku, Nagoya 453-8511, Japan. Tel. +81-52-481-5111 Fax. +81-52-482-7733. E-mail akirayokoi@nagoya-1st.jrc.or.jp.

(c) Ivyspring International Publisher. This is an open-access article distributed under the terms of the Creative Commons License (http://creativecommons.org/ licenses/by-nc-nd/3.0/). Reproduction is permitted for personal, noncommercial use, provided that the article is in whole, unmodified, and properly cited.

Received: 2012.06.01; Accepted: 2012.08.06; Published: 2012.08.15

\begin{abstract}
Aim: To validate a previously developed prediction model for vaginal birth after cesarean (VBAC) using a Japanese cohort.

Methods: We performed a cohort study of all term pregnant women with a vertex position, singleton gestation, and one prior low transverse cesarean delivery attempting a trial of labor between April 1985 and March 20I0. Variables necessary for the prediction of successful VBAC were maternal age, pre-pregnancy body mass index, ethnicity, prior vaginal delivery, prior VBAC, and indication for prior cesarean delivery. They were extracted from medical records and put into the formula that calculates an individual woman's predicted VBAC success rate. The predicted rates were then partitioned into deciles and compared with the actual VBAC rates. The predictive ability of the model was assessed with a receiver operating characteristic and the area under the curve (AUC) was determined.

Results: Seven hundred and twenty-five women who met the inclusion criteria had complete data available, of which 664 (91.6\%) had VBAC. The predicted probability of VBAC, as calculated by the regression equation, was significantly higher in those who had a successful trial of labor (median 80. I\%, interquartile range 7I.5-88.7) than those who did not (median 69.4\%, interquartile range 59.9-78.9, $P<0.00 \mathrm{I}$ ). The predictive model had AUC of 0.80 , which was comparative to the originally described one. When the predicted rates were each deciles of over $70 \%$, the actual success rates were more than $90 \%$.

Conclusion: The previously published prediction model for VBAC developed in the USA is also available to Japanese women.
\end{abstract}

Key words: vaginal birth after cesarean, trial of labor after cesarean, prediction model, Japanese, validation.

\section{Introduction}

The increasing cesarean section rate is a global issue in industrialized countries. Cesarean delivery rates in the United States have now reached their highest levels ever, accounting for 32.3\% in 2008 (1). The same is equally true of Japan; the rate stood at $19.8-34.1 \%$ around the same time (2-4). The recent increase in the total cesarean deliveries has been caused by a steady increase in primary cesarean sections and a sharp and persistent decrease in vaginal birth after cesarean (VBAC) (1). There is a commonly-held view that operative complications such as hysterectomy, blood transfusion, and dense adhesions 
increases with multiple cesarean sections $(5,6)$. In addition, placenta previa or accera is more often seen as the number of prior cesarean deliveries increases (5-7). Furthermore, increase in cesarean sections will lead to escalation of medical costs in the future, which may bring worrisome issues on medical economy.

The National Institutes of Health held a Consensus Development Conference in March 2010 to examine the evidence on maternal and neonatal outcomes in trial of labor after cesarean (TOLAC) and to reach evidence-based consensus statement, of which conclusion is that TOLAC remains a reasonable option for many women with a prior cesarean delivery (8). In an effort to decrease the rate of cesarean delivery, the American College of Obstetricians and Gynecologists (ACOG) has recommended that most pregnant women with a single previous low transverse cesarean delivery be counseled about VBAC and be offered a trial of labor (9). A key aspect in the counseling process involves providing the woman with her individual chance of success as well as a discussion about the maternal and neonatal risks and benefits associated with TOLAC.

Recently, Grobman et al (10) has developed a model that predicts the probability of successful TOLAC for Caucasians, Hispanics, and African Americans in the United States of America (USA). The probability can be calculated by using an easy-to-use calculator found at http://www.bsc.gwu.edu/ $\mathrm{mfmu} /$ vagbirth.html. The variables for calculation consist of maternal age, pre-pregnancy body mass index (BMI), ethnicity, prior vaginal delivery, prior VBAC, and indication for prior cesarean delivery that can be obtained at the first prenatal visit, then allowing obstetricians to provide a woman desiring a trial of labor with her individual chance of success early in gestation.

Unfortunately, no validation has been conducted in the other countries and ethnicities except for Caucasians, Hispanics, and African Americans in the USA. The objective of this study was to validate the VBAC prediction model and to test its accuracy in Japanese cohort.

\section{Materials and Methods}

Japanese Red Cross Nagoya Daiichi Hospital, Nagoya, Japan is a tertiary referral center with about 1400 deliveries per year. In this study, all Japanese pregnant women with a vertex position, singleton gestation at term (more than 36 weeks), and a single prior low transverse cesarean delivery who attempted a trial of labor between April 1984 and March 2010 in the hospital were identified. We picked up only women who were ahead of the onset of labor. Medical records were reviewed and pertinent data were collected. This study was approved by the Institutional Ethical Committee of Japanese Red Cross Nagoya Daiichi Hospital. As reported by Grobman et al (10), the prediction model includes maternal characteristics that can be obtained at the first prenatal visit: maternal age, pre-pregnancy BMI $\left(\mathrm{kg} / \mathrm{m}^{2}\right)$, ethnicity, any prior vaginal delivery, prior VBAC, and indication of prior cesarean delivery. The data from individual patients were incorporated into the reported regression formula, and the predicted success rate of TOLAC for each woman was calculated.

The predicted VBAC scores at the level of the study population then were partitioned into 10 decile groups (e.g. $0-10 \%, 10-20 \%$ ). In each of the deciles, the proportion of women with a successful TOLAC was calculated. These proportions represent the actual or observed rates of VBAC.

The predictive ability of the model was assessed with a receiver operating characteristic (ROC). The area under the curve (AUC) was determined by the trapezoidal rule (empirical or nonparametric method) (11).

The significant differences and trends in variables across the successful and failed groups were assessed with the Student's t-test. All analyses and figures were performed using SPSS version 17.0 for Windows (IBM, New York, USA). Values of $P<0.05$ were considered statistically significant.

\section{Results}

During the study period, 1421women had a past history of prior cesarean section and 725 women tried VBAC; the rate of TOLAC was $51.0 \%$. Seven hundred and twenty-five women met the inclusion criteria and complete data were available. The women' characteristics are summarized in Table 1. A total of 664 patients had VBAC, corresponding to a success rate of $91.5 \%$. The predicted probability of VBAC, as calculated by the regression equation, was significantly higher in those who had a successful trial of labor (average $80.1 \%$, interquartile range $71.5-88.7$ ) than in those who did not (average $69.4 \%$, interquartile range $59.9-78.9, P<0.001)$.

The efficacy of the prediction model for VBAC in Japanese women was presented as the ROC, and the AUC was 0.81 (95\% CI 0.75- 0.87, P<0.001), which was almost consistent with the AUC of 0.75 reported in the development of the prediction model (10).

The predicted VBAC success rates were partitioned into deciles and were compared with the observed VBAC rates (Table 2). The actual VBAC rates did not differ from the predicted ones when the predicted success rates were less than $70 \%$. When the 
predicted rates were over $70 \%$, the actual success rates were more than $90 \%$. In addition, the reasons for discontinuing TOLAC were shown in Table 3. Hereby, there was one woman developed uterine rupture during TOLAC.

Table I. Characteristics of patients attempting a trial of labor.

\begin{tabular}{|c|c|c|c|c|}
\hline Variable & $\begin{array}{l}\text { Total } \\
(\mathrm{n}=725)\end{array}$ & $\begin{array}{l}\text { Success } \\
(\mathrm{n}=664)\end{array}$ & $\begin{array}{l}\text { Failed } \\
(n=61)\end{array}$ & $p$ value $^{*}$ \\
\hline Maternal age (y) & $30.5 \pm 4.1$ & $30.4 \pm 4.1$ & $31.4 \pm 4.0$ & 0.08 \\
\hline $\begin{array}{l}\text { Pre-pregnancy BMI } \\
\left(\mathrm{kg} / \mathrm{m}^{2}\right)\end{array}$ & $21.5 \pm 3.3$ & $21.4 \pm 3.1$ & $22.6 \pm 4.6$ & 0.05 \\
\hline height $(\mathrm{cm})$ & $156.3 \pm 5.1$ & $156.2 \pm 7.9$ & $\begin{array}{l}152.5 \pm \\
18.8\end{array}$ & 0.13 \\
\hline weight (kg) & $52.7 \pm 8.9$ & $52.5 \pm 8.4$ & $54.6 \pm 8.4$ & 0.25 \\
\hline $\begin{array}{l}\text { Any prior vaginal } \\
\text { delivery }\end{array}$ & $127(17.5)$ & 124(18.7) & $3(4.9)$ & $<0.01$ \\
\hline Prior VBAC & $80(11.0)$ & $80(12.0)$ & $0(0.0)$ & $<0.01$ \\
\hline \multicolumn{5}{|l|}{$\begin{array}{l}\text { Indication of prior } \\
C D\end{array}$} \\
\hline $\begin{array}{l}\text { Arrest of dilation or } \\
\text { descent }\end{array}$ & $177(24.4)$ & $136(76.8)$ & $41(23.3)$ & $<0.01$ \\
\hline $\begin{array}{l}\text { Non reassuring fetal } \\
\text { status }\end{array}$ & $94(13.0)$ & $84(12.7)$ & $10(16.4)$ & 0.40 \\
\hline Breech presentation & $182(25.1)$ & $177(26.7)$ & $5(8.2)$ & $<0.01$ \\
\hline Others & $272(37.5)$ & - & - & - \\
\hline
\end{tabular}

BMI, body mass index; VBAC, vaginal birth after cesarean; CD, cesarean delivery. Data are mean \pm standard deviation or $n(\%) .{ }^{*} ; \chi^{2}$ test or Student-t test

Table 2. Observed success rate of VBAC according to the predicted rate.

\begin{tabular}{llll}
\hline \multicolumn{3}{c}{ VBAC } \\
\hline $\begin{array}{l}\text { Predicted success rate } \\
(\%)\end{array}$ & $\mathrm{N}$ & $\mathrm{N}$ & $\begin{array}{l}\text { Observed } \\
\text { success } \\
\text { rate }(\%)\end{array}$ \\
\hline $0-10$ & & 0 & $\mathrm{n} / \mathrm{a}$ \\
$10-20$ & 0 & 0 & $\mathrm{n} / \mathrm{a}$ \\
$20-30$ & 0 & 0 & $\mathrm{n} / \mathrm{a}$ \\
$30-40$ & 0 & 0 & $\mathrm{n} / \mathrm{a}$ \\
$40-50$ & 0 & 2 & 67 \\
$50-60$ & 3 & 9 & 53 \\
$60-70$ & 17 & 58 & 70 \\
$70-80$ & 83 & 258 & 93 \\
$80-90$ & 276 & 239 & 98
\end{tabular}

VBAC, vaginal birth after cesarean; $N$, number of observed; $n / a$, not available.
Table 3. Reasons for discontinuing TOLAC.

\begin{tabular}{ll}
\hline Reasons & $\mathrm{N}$ \\
\hline Arrest of dilation or descent & 38 \\
Non reassuring fetal status & 18 \\
Uterine rupture & 1 \\
Others & 4 \\
\hline
\end{tabular}

\section{Discussion}

Grothmann et al (10) developed the prediction model using the data from the people living in the USA. The model provides important information that can be used to provide individual and patient-specific success rates of TOLAC. Afterward, Costantine et al (12) confirmed its availability in an entirely different cohort in the USA from that the original prediction model was worked out. However, no validation has been done for Japanese women. Japanese people have different features from Caucasians, Hispanics, and African Americans in the USA. Physical type is probably one of the most distinguished differences. It is reflected by the findings that the average of pre-pregnancy BMI was 21.5 in the present study compared to 26.4 and 27.5 in the study by Grothmann et al. (10) and Costantine et al. (12), respectively. BMI is one of the considerable factors for vaginal delivery because the cesarean rate increases with the pre-pregnancy BMI (13). Therefore, it is necessary to evaluate the prediction model for VBAC beforehand when we Japanese utilize it.

It was unexpected that VBAC rate, which means successful TOLAC, was $91.5 \%$ in the presenting study and much higher than the ones of $52.2 \%-74.4 \%$ in the USA $(10,12,14)$. Furthermore, the achieved success rates were more than $90 \%$ in any deciles above $50 \%$ predicted success in our study. In contrast, the point estimates of the observed VBAC rates were approximately $10-20 \%$ lower than the predicted rates when the predicted rate was at least $50 \%$ in the study by Costatine et al. (12). These discrepancies may be due to the different cohort undergoing TOLAC between Japan and the USA. Besides the ethnicity, the rate of any prior vaginal delivery, the prior VBAC, and the recurring indication of prior cesarean delivery were appreciably different. Those values in our study and the one by Costantine et al. (12) were $17.5 \%, 11.0 \%$, $24.4 \%$ and $49.4 \%, 33.3 \%, 39.6 \%$, respectively. Regarding the rate of TOLAC, it was $51.0 \%$ in the presenting study and was not different from the ones seen in the USA (14). Anyhow, these differences in the background are likely to affect the success rate of TOLAC between Japan and the USA. 
Although the prediction model is verified to be useful in Japanese pregnant women in the present study, it might be difficult to increase the success rate of TOLAC in whole Japan since it depends on variable factors including medical reasons. First, treatment policy is different among obstetricians, which may lead to unsuccessful VBAC. For example, criterion for cesarean section against the abnormal fetal heart rate monitoring is variant albeit the identical abnormal pattern. Each obstetrician has his own policy on the duration until the cesarean section after rupture of the membranes. Second, settings of TOLAC such as hospital volume and type may affect the maternal outcomes. A positive relation between high-volume obstetric units and better outcomes of VBAC has been reported $(15,16)$. Third, geographical difference of whether the area is urban of rural has an influence on VBAC. In the USA, VBAC rates were lower in rural hospitals than in urban hospitals (17). VBAC deliveries declined more rapidly at rural hospitals after 1999 when the ACOG revised the guideline for VBAC to place greater emphasis on safety. No data has been reported in Japan, but it is likely the same. Based on the above reasons, even women undergoing TOLAC with high-predicted success rate have a number of issues to get to the goal of vaginal delivery.

Nevertheless, the presenting study is meaningful in that the prediction model for successful VBAC is a guidepost to women with prior cesarean section. The model gives women contemplating TOLAC individual chances of success to help them reach a more informed decision. The predicted success rate is easily calculated and indicated at the first visit, giving a great deal of time to consider. Information with the numerical value is helpful for both obstetricians and women considering TOLAC.

Finally, it should be emphasized that obstetricians do not use the predicted success rate as a cutoff above or below which they will or will not offer a TOLAC. Women themselves, rather than policymakers, providers, insurance carriers, or hospitals, should determine delivery approach (18).

In conclusion, the prediction model developed by Grobman et al. (10) is applicable to Japanese women.

\section{Acknowledgments}

We thank medical staff in Department of Obstetrics and Gynecology, Japanese Red Cross Nagoya Daiichi Hospital for their support.

\section{Competing Interests}

The authors have declared that no competing interest exists.

\section{References}

1. Martin JA, Hamilton BE, Sutton PD, Ventura SJ, Mathews TJ, Osterman MJK. Births: final data for 2008. Natl Vital Stat Rep 2010; 59: 1-72.

2. Niino $Y$. The increasing cesarean rate globally and what we can do about it. Biosci Trends 2011; 5: 139-150.

3. Lumbiganon P, Laopaiboon M, Glümezoglu AM et al. Method of delivery and pregnancy outcomes in Asia: the WHO global survey on maternal and perinatal health 2007-08. Lancet 2010; 375: 490-499.

4. [No authors listed]. Report of perinatal committee. Acta Obstet Gynaecol Jpn 2011; 63: 1318-1335.

5. Andrews D, Humphries G. After a cesarean...What's a birth professionals to do? J Perinat Educ 2010; 19: 11-15

6. Clark EA, Silver RM. Long-term maternal mormidity associated with repeat cesarean delivery. Am J Obstet Gynecol 2011; 205: S2-10.

7. Gurol-Urganci L, Cromwell DA, Edozien LC et al. Risk of placenta previa in second birth after first birth cesarean section: a population-based study and meta-analysis. BMC Pregnancy Childbirth 2011; 11: 95.

8. National Institutes of Health Consensus Development Conference Statement: Vaginal Birth After Cesarean: New Insights March 8-10, 2010. Obstet Gynecol 2010; 115: 1279-1295.

9. ACOG Practice bulletin no. 115: Vaginal birth after previous cesarean delivery. American College of Obstetricians and Gynecologists. Obstet Gynecol 2010; 116: 450-463.

10. Grobman WA, Lai Y, Landon MB et al. Development of a Nomogram for Prediction of Vaginal Birth After Cesarean Delivery. Obstet Gynecol 2007; 109: 806-812.

11. Hanley JA, McNeil BJ. The meaning and use of the area under a receiver operating characteristic (ROC) curve. Radiology 1982; 143: 29-36.

12. Constantine MM, Fox K, Byers BD et al. Validation of the prediction model for success of vaginal birth after cesarean delivery. Obstet Gynecol 2009; 114: 1029-1033.

13. Mamun AA, Callaway LK, O'Callaghan MJ et al. Associations of maternal pre-pregnancy obesity and excess pregnancy weight gains with adverse pregnancy outcomes and length of hospital stay. BMC Pregnancy Childbirth 2011; 11: 62.

14. Grobman WA, Lai $Y$, Landon MB et al. The change in the rate of vaginal birth after caesarean section. Paediatr Perinat Epidemiol 2011; 25: 37-43.

15. Wen SW, Rusen ID, Walker M et al. Comparison of maternal mortality and morbidity between trial of labor and elective cesarean section among women with previous cesarean delivery. Am J Obstet Gynecol 2004, 191 : 1263-1269.

16. McMahon MJ, Luther ER, Bowes WA Jr, Olshan AF. Comparison of a trial of labor with an elective second cesarean section. N Engl J Med 1996; 335: 689-695.

17. Sieck CC. Vaginal birth after cesarean section: a comparison of rural and metropolitan rates in Oklahoma. J Okla State Med Assoc 1997; 90: 444-449.

18. Lyerly AD, Little MO. Toward an ethically responsible approach to vaginal birth after cerasean. Semin Perinatol 2010; 34: 337-344. 